\title{
Applications of geoinformatics for the prevention and mitigation of natural hazards
}

\author{
Silas Michaelides ${ }^{1,2}$
}

(C) Springer Science+Business Media Dordrecht 2016

This Special Issue of Natural Hazards is entitled "Applications of Geoinformatics for the Prevention and Mitigation of Natural Hazards." It encompasses selected papers that were presented at the "Third International Conference on Remote Sensing and Geoinformation of Environment-RSCy2015" that was held in Cyprus from March, 16 to 19, 2015. The Special Issue comprises thirteen papers which cover a wide spectrum of geoinformatics applications.

I am very glad to have been invited to act as the Guest Editor for this Special Issue of Natural Hazards. I consider it a privilege and an honor to closely collaborate with so many authors in order to deliver this volume after a strict anonymous review process.

The first paper in this volume by Agapiou et al. forms an attempt to assess the risk of natural and anthropogenic hazards for cultural heritage in Cyprus by integrating multitemporal GIS and earth observation analyses based on homogeneous clustering of the local monuments under consideration. The proposed clustering of monuments is based on a variety of parameters, taking into consideration characteristics of their immediate environment. For each one of the five clusters of monuments studied, an analytical hierarchy process (AHP) method is followed in order to address the individual and unique characteristics of the monuments and sites. Subsequently, the weight factors from these clusters were interpolated to the whole district, prior to the application of the overall AHP risk assessment.

Fiorani et al. report on the direct measurement of carbon dioxide in a volcanic plume as retrieved by a volcanic lidar, thus showing the high potential of laser remote sensing in early detection of volcanic hazard. Anomalous releases of carbon dioxide precede volcanic eruptions. The development of the volcanic lidars has been undertaken at the Italian National Agency for New Technologies, Energy and Sustainable Economic Development. Lidar profiling allows the scanning of volcanic plumes remotely in a fast and continuous

Silas Michaelides

silas.michaelides@cut.ac.cy

1 Cyprus University of Technology, Limassol, Cyprus

2 The Cyprus Institute, Nicosia, Cyprus 
way, and with high spatial and temporal resolution. Using a differential absorption lidar instrument, the gas emitted by Pozzuoli Solfatara (Naples, Italy) and Stromboli Volcano (Sicily, Italy) was scanned during field campaigns. Carbon dioxide concentration maps were retrieved remotely in a few minutes in the respective crater areas.

Franci et al. focus on the exploitation of very-high-resolution (VHR) satellite imagery coupled with multi-criteria analysis (MCA) to produce flood hazard maps. The MCA methodology was performed selecting five flood-causing factors: slope, distance to channels, drainage texture, geology and land cover. Among MCA methods, the analytic hierarchy process technique was chosen to derive the weight of each criterion in the computation of the flood hazard index (FHI). The required information layers were obtained by processing a VHR GeoEye-1 image and a digital elevation model. Using the FHI, the study area was finally classified into seven hazard categories, ranging from very low to very high in order to generate an easily readable map.

Katsanos et al. carried out their study by using a daily precipitation database for the island of Cyprus for a period of 30 years. A number of climatic indices for precipitation are calculated using the recently available CHIRPS dataset, on high spatial $\left(0.05^{\circ}\right)$ and temporal (daily) resolution. The same parameters for the same time period (1981-2010) are then calculated using a dense network of rain gauges. The results show a quite promising performance regarding indices related to daily precipitation thresholds, resulting in high correlation scores. In the case of indices referring to number of days, it seems that the results are ambiguous, with medium or no correlation, probably related to the criteria used for the identification of a wet (rainy) day on the CHIRPS dataset.

Kourgialas et al. present a dynamic GIS modeling approach that incorporates geoinformatic techniques, historical meteorological data and measurements, in order to estimate soil erosion risk in intensively cultivated regions. The proposed GIS-based modeling approach includes the estimation of soil erosion rates due to surface water flow under current and future climate change scenarios. The soil erosion was estimated using the universal soil loss equation (RUSLE), and the proposed soil erosion model was validated using field measurements at different sites.

Mettas et al. describe a range of different remote sensing methodologies that can be used to monitor asphalt road pavements. This is complemented with the use of field spectroscopy for the examination of asphalt pavements of varying age and conditions. The results of the study found spectral differences regarding asphalt defects, such as physical cracking, patched cracking and polishing. These differences were examined through "inband" simulation analysis of the Landsat 7 ETM+ sensor, using appropriate relative spectral response filters. The authors conclude that the ratio band 5: Band 1 can be used to distinguish asphalt pavements of different date of construction and condition.

Oikonomou et al. demonstrate how ionospheric total electron content (TEC) variations prior to two large earthquakes in Nepal $(M=7.8)$ and Chile $(M=8.3)$ in 2015 were analyzed using measurements from the global navigation satellite system (GNSS) network. The aim was to detect possible ionospheric anomalies associated with these seismic events and describe their main features, by applying statistical and spectral analysis. It was shown that abnormal TEC variations appeared few days up to few hours before the events lasting up to $8 \mathrm{~h}$, whereas intensified TEC wave-like oscillations with periods 20 and 2-5 min were also identified that could be linked to the impending earthquakes. An unusual modification of the equatorial ionospheric anomaly 5 days before the main shock was also detected.

Papaioannou et al. present an innovative approach in the investigation of complex landscapes for hydraulic modeling applications by using a terrestrial laser scanner (TLS) 
that can lead to a high-resolution digital elevation model (DEM). Another notable factor in flood modeling is the selection of the hydrodynamic model, especially in complex riverine topographies that can influence the accuracy of flood inundation area and mapping. The paper uses different types of hydraulic-hydrodynamic modeling approaches and several types of river and riparian area spatial resolution for the implementation of a sensitivity analysis for floodplain mapping and flood inundation modeling process at ungauged watersheds. Results provided from contingency table analysis indicate the sensitivity of floodplain modeling on the DEM spatial resolution and the hydraulic modeling approach.

Partsinevelos et al. present an operational low-cost rockfall monitoring and alerting system. The system integrates measurements from a multi-channel seismograph and commercial cameras as the primary equipment for event detection. A series of algorithms analyze these measurements independently in order to reduce alarms originating by surrounding noise and sources other than rockfall events. The detection methodology employs two different sets of algorithms. Training and validation of the proposed approach was performed through field tests that involved manually induced rockfall events and recording of sources (i.e., passing car, walking people) that may cause a false alarm.

Land subsidence which is a common natural hazard striking extensive areas worldwide is studied by Raspini et al. Within the framework of the ESAGMES Terrafirma project (2003-2012), a series of products, based on the integration of earth observation satellite synthetic aperture radar interferometry (InSAR) technologies and in situ data, has been implemented. The paper covers three case studies that have been selected with the purpose of showing the essential contribution of interferometric data during the main activities that must be tackled when dealing with geohazard investigations (i.e., mapping, monitoring and modeling). These three case studies were meant to be representative of the suite of services delivered by the Terrafirma project to specific end users.

Tantele et al. elaborate on the preparation of a corrosion risk map as a result of carbonation-induced corrosion of the steel reinforcement that is the major deterioration factor of infrastructures in urban areas. The rate of deterioration due to carbonation varies between different areas. Field measurements can quantify this carbonation progress for specific structures and areas. However, the scattered nature of individual field data offers little information to be considered for the assessment of existing structures or the design of new structures. This study aims to bridge this gap and show that individual field data can be combined to characterize an area by using GIS mapping tools. A generated map can depict the variability of carbonation progress with the geographical location.

In their contribution, Velegrakis et al. present the results of their study on the shoreline variability of a natural perched urban beach. Variability of a shoreline in Greece was monitored in high spatiotemporal resolution using time series of coastal video images and a novel, fully automated 2-D shoreline detection algorithm. Ten-month video monitoring showed that cross-shore shoreline change was, in some areas, up to $8 \mathrm{~m}$ with adjacent sections of the shoreline showing contrasting patterns of beach loss or gain. Variability increased in spring/early summer and stabilized until the end of the summer when partial beach recovery commenced. Correlation of the patterns of beach change with wave forcing (as recorded at an offshore wave buoy) is not straightforward.

The last paper in this issue is by Zanchetta et al. who put forward a method for monitoring sensitive areas like oases which are threatened by climatic variations and human activities catalyzing the desertification processes. In this paper, a remote sensing method that has been widely used for vegetated areas is adapted to study dry regions. The method consists of a combination of the change vector analysis (CVA) and the tasselled cap (TC) transform. To adapt it to dryland conditions, a new set of parameters for the TC 
transform is calculated for the Landsat 8 OLI system. The new TC parameters are tested in the analysis of the surface change in Azraq Oasis, Jordan, over a span time of 30 years (1984-2013). Results show that the chosen technique is able to detect the expected change on the surface, consistent with photointerpretation and historical information available.

As this Special Issue covers a wide spectrum of applications of geoinformatics related to various Natural Hazards, I expect that the readers will appreciate the importance of the benefits that can be reached for their prevention and mitigation. I also believe that the articles presented in this volume will form a valuable reference to researchers, academics, practitioners and policy makers.

As the Guest Editor of this Special Issue of Natural Hazards, I would like to thank all the authors for their contributions to this volume and their adherence to the strict reviewing rules and procedures. I also wish to express my sincere appreciation to the international panel of distinguished reviewers for making available their expertise in the effort to achieve the highest scientific value of this volume, especially under a tight time schedule. I wish to express my special thanks to the editorial team and in particular to Prof. Tad Murty, the editor-in-chief of Natural Hazards, for their valuable support during all stages of producing this volume. I also extend my thanks to the members of the staff of the publisher for their professional technical assistance. 